\title{
Assessment of carotid-intima media thickness in patients with epilepsy receiving antiepileptic drugs
}

\author{
๑Dehtap Kocatürk ${ }^{1}$, @Özcan Kocatürk ${ }^{2}$ \\ ${ }^{1}$ Balıkesir University, Department of Neurology, Balıkesir, Turkey \\ ${ }^{2}$ Balıkesir Atatürk City Hospital, Department of Neurology, Balıkesir, Turkey
}

Cite this article as: Kocatürk M, Kocatürk Ö. Assessment of carotid-intima media thickness in patients with epilepsy receiving antiepileptic drugs. J Health Sci Med 2022; 5(1): 234-238.

\begin{abstract}
Aim: This study aims to investigate whether there is an increase in carotid-intima media thickness (C-IMT) in epilepsy patients using antiepileptic drugs when compared to the normal control group and whether there is a difference when we group them as monotherapy and polytherapy.

Material and Method: 68 people were included in the prospective cross-sectional study. 38 epilepsy and 30 healthy control groups that were matched in terms of demographic characteristics

Results: C-IMT was statistically higher in the epilepsy group $(\mathrm{p}<0.001)$. When carotid USG results are analyzed as control, monotherapy, and polytherapy, there was no difference between control and monotherapy, there was statistical significance between the control group and polytherapy $(\mathrm{p}<0.001)$, and between monotherapy and polytherapy (p:0.010)

Conclusions: According to our study result, polytherapy should be avoided as much as possible, especially in patients with vascular risk factors.
\end{abstract}

Keywords: Carotid-intima media thickness, epilepsy, monotherapy-polytherapy

\section{INTRODUCTION}

Epilepsy affects 65 million people worldwide and is among the most common neurological diseases (1). Treatment is based on long-term anti-epileptic drugs (AEDs) and the use of these drugs is sometimes lifelong. Treatment aims to try to ensure seizure-freeness with drugs while minimizing the side effects of these drugs. Therefore, the physician should have a good knowledge of the side-effect profile of the drugs and know-how to manage the side-effects when they develop.

Long-term use of AEDs is associated with chronic adverse events such as metabolic and endocrine disorders, organ toxicity, cognitive dysfunction, and psychiatric problems (2-5). Studies have reported that patients with epilepsy have earlier deaths from stroke and ischemic heart disease compared to the general population $(6,7)$. Although the reason for this relationship cannot be fully explained, it is thought that drugs may be responsible for this situation along with the disease itself. One of the reasons why mortality rates are higher than the general population may be susceptibility to atherosclerosis.
The impact of AEDs on the development of atherosclerosis has been discussed for a long time. The role of drugs in the pathogenesis of atherosclerosis has not been fully elucidated. Studies are showing that especially old generation antiepileptic drugs can cause dyslipidemia and hyperhomocysteinemia, which may lead to the development of atherosclerosis $(8,9)$. In addition, inflammation marker CRP, which is closely related to atherosclerosis, and oxidative stress markers were detected in epilepsy patients using AED (10). There is also one opinion that atherosclerosis may be involved in the etiology of chronic cognitive dysfunction observed in epilepsy patients (11).

Carotid-intima media thickness (C-IMT) is an early marker of atherosclerosis and its increase is a predictor of future vascular events. Although they have controversial results, studies are showing that C-IMT increase in epilepsy patients $(12,13)$. In this study, we aim to investigate whether there is an increase in C-IMT in epilepsy patients using antiepileptic drugs when compared to the normal control group and whether there is a difference when we group them as monotherapy and polytherapy. 


\section{MATERIAL AND METHOD}

The study was approved by the Harran University Faculty of Medicine Ethics Committee (Date: 08.07.2019, Decision No: 07-13). All procedures were carried out in accordance with the ethical rules and the principles of the Declaration of Helsinki.

This cross-sectional study included 68 subjects, 38 epilepsy who were taking one or more antiepileptic drugs, and 30 healthy control. The study was conducted from June 2019 to September 2019 at the Neurology Department of Harran University Hospital. Exclusion criteria are including patients under 18 and over 50, body mass index above 35, hyperlipidemia, known heart disease, hypertension, diabetes mellitus, oral contraceptive usage, history of the previous stroke, multiple sclerosis, and inflammatory disease. Patients were also excluded from the study if the epilepsy was caused by metabolic or neurodegenerative diseases and taking any medication or vitamin preparations other than antiepileptic drugs. Patients were selected from those diagnosed as having epilepsy according to the International League Against Epilepsy (ILEA) 2017 criteria and who were using one or more antiepileptic drugs in treatment (14). The diagnosis of epilepsy made by an experienced neurologist (M.K.), was based on the clinical history, including the response to medication, seizure description, and electroencephalography, and magnetic resonance imaging result. The control group consisted of individuals of the same age, sex, and BMI without a history of epilepsy. The physical and neurological examinations of the control group were normal. Written informed consent was obtained from the patients before being included in the study. Body mass index (BMI) was calculated as weight $(\mathrm{kg}) /$ height $\left(\mathrm{m}^{2}\right)$. Hypertension was defined as systolic blood pressure $\geq 140 \mathrm{mmHg}$, diastolic blood pressure $90 \mathrm{mmHg}$, and/or taking antihypertensive medication, and/or a history of diagnosed hypertension. Diabetes mellitus was defined as fasting serum glucose $\geq 126 \mathrm{mg} / \mathrm{dL}(7 \mathrm{mmol} / \mathrm{L})$, non-fasting glucose $\geq 200 \mathrm{mg} /$ dL (11.1 mmol/L), use of diabetic medications, or a previously established diagnosis. Hyperlipidemia was defined as low-density lipoprotein $>160 \mathrm{mg} / \mathrm{dL}$, and/or taking a lipid-lowering agent.

Bilateral carotid arteries USG examination was done by the same neurologist (Ö.K. with 7 years of US experience and were blinded to epilepsy diagnosis and clinical data). The examinations were performed using the LOGIQ V5 (GE Medical Systems, Wuxi, China) with the same linear-array transducers. In the supine position, bilateral common and internal carotid arteries were examined for the presence of atherosclerotic plaques, using grayscale and color Doppler modes. C-IMT was defined as the thickness of the hypoechoic layer between the vessel lumen and hyperechoic adventitia. C-IMT was measured in the posterior walls of both left and right CCA at $1 \mathrm{~cm}$ below the carotid bifurcation outside the areas of plaque and the average of two values (right wall and left wall) was recorded as C-IMT mean.

\section{Statistical Analyses}

The parameters were analyzed using SPSS for Windows ver. 23.0. Continuous variables are expressed as the mean \pm standard deviation and categorical variables as numbers and percentages. The Mann-Whitney $U$ test was used to assess the differences between continuous variables in two groups. The Kruskal-Wallis test was used to compare more than two independent groups. Correlation between continuous variables was evaluated with the Spearman correlation test. The chi-square test was used to analyze categorical parameters and $\mathrm{p}<0.05$ was considered statistically significant.

\section{RESULTS}

This study compared healthy control $(\mathrm{n}=30$; mean age $27.57 \pm 6.10$ years) and epilepsy $(n=38$; mean age $25.58 \pm 7.61$ years) groups. There were no significant intergroup differences in age, percentage of sex, serum lipid level, or BMI (Table 1).

Table 1. Demographic and clinical findings of the epilepsy patient and control groups

\begin{tabular}{|lccc|}
\hline & Epilepsy n :38 & Control n: 30 & p \\
\hline C-IMT & $0.46( \pm 0.08)$ & $0.37( \pm 0.04)$ & 0.000 \\
Age (years) & $25.58( \pm 7.61)$ & $27.57( \pm 6.10)$ & 0.248 \\
Female, $\mathrm{n}(\%)$ & $24(55.8)$ & $19(44.2)$ & 0.595 \\
BMI $\left(\mathrm{kg} / \mathrm{m}^{2}\right)$ & $23.29( \pm 4.38)$ & $24.15( \pm 3.52)$ & 0.381 \\
Epilepsy type (ILEA-2017) & & & \\
Focal onset & $24(63.2)$ & & \\
Generalized onset & $10(26.3)$ & & \\
Unknown Onset & $4(10.4)$ & & \\
Seizure etiology, n $(\%)$ & & & \\
Structural & $12(31.6)$ & & \\
Genetic & $10(26.3)$ & & \\
Unknown & $16(42.1)$ & & \\
Disease duration (years) & $7.84( \pm 5.82)$ & & \\
Number of AED & & & \\
Monotherapy & $16(42.1 \%)$ & & \\
Polytherapy & $22(57.9 \%)$ & & \\
Serum lipid level (mg) & & & \\
LDL cholesterol & $107.73( \pm 16.45)$ & $99.62( \pm 14.20)$ & 0.201 \\
HDL cholesterol & $60.39( \pm 15.11)$ & $53.82( \pm 14.60)$ & 0.074 \\
Triglyceride & $100.59( \pm 36.55)$ & $109.08(33.28)$ & 0.521 \\
\hline C-IMT: Carotid intima media thickness, BMI: Body Mass Index & \\
AED: Anti Epileptic Drug, LDL:Low Density Lipoprotein HDL: High Density \\
Lipoprotein
\end{tabular}

The patients were diagnosed as having focal epilepsy in $24(63.2 \%)$, generalized epilepsy in $10(26.3 \%)$ cases, and unknown in $4(10.4 \%)$. Of the patients in the study group, $16(42.1 \%)$ were receiving monotherapy and $22(57.9 \%)$ 
were receiving polytherapy. Eighteen of the patients in the polytherapy group were taking double antiepileptic, and 4 were taking triple antiepileptic. Considering the etiology of seizures, 12 (31.6\%) patients are structural, $10(16.3 \%)$ patients are genetic, and the etiology of $16(42.1 \%)$ patients is unknown.

When the study population was analyzed as monotherapy $(n=16$; mean age $23.88 \pm 7.32$ years), polytherapy $(n=22$; mean age $26.82 \pm 7.73$ years), and control groups $(n=30$; mean age $27.57 \pm 6.10$ years), there was no difference between the groups in terms of age, gender, BMI and serum lipid levels (Table 2).

When carotid ultrasound findings were compared between epilepsy and control groups, C-IMT was statistically higher in the epilepsy group $(\mathrm{p}<0.001)$ (Table 1). When carotid USG results are analyzed as control, monotherapy, and polytherapy, there was no difference between control and monotherapy, there was statistical significance between the control group and polytherapy $(\mathrm{p}<0.001)$, and between monotherapy and polytherapy (p:0.010) (Table 2). Distribution of antiepileptic drugs used by the patients were showed in Table 3. Figure 1 showed that there was no relationship between the duration of epilepsy and the increase in C-IMT.

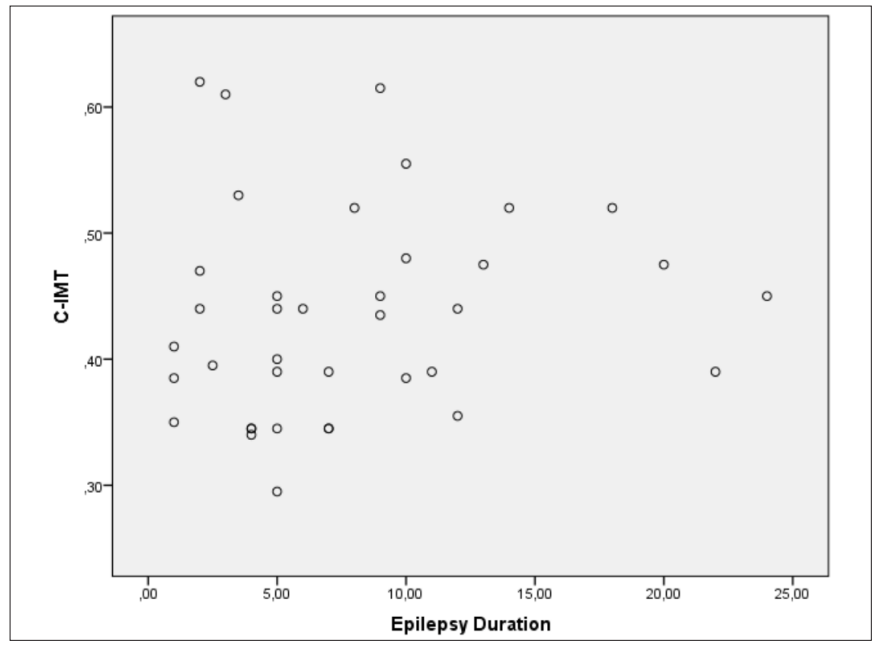

Figure 1. Correlation between epilepsy duration and C-IMT (p:0.217). C-IMT: Carotid-intima-media thickness

\begin{tabular}{|lccc|}
\hline \multicolumn{4}{|c|}{ Table 3. Distribution of antiepileptic drugs used by the patients } \\
\hline & Monotherapy (n) & Polytherapy(n) & Total (n) \\
\hline Levetirecetam & 6 & 13 & 19 \\
Lamotrigine & 2 & 11 & 13 \\
Valproic acid & 5 & 9 & 14 \\
Carbamazepine & 3 & 10 & 13 \\
Topiramate & - & 2 & 2 \\
\hline
\end{tabular}

\section{DISCUSSION}

C-IMT measurement is a noninvasive ultrasonographic method and is applied easily and inexpensively (12). Its increase is indicative of subclinical atherosclerosis and is associated with many vascular events, including cardiovascular and cerebrovascular accidents (15). Although there are studies evaluating whether there is an increase in C-IMT in epilepsy patients using antiepileptic drugs, according to our knowledge, there is no study evaluating the increase in C-IMT in adult patients according to polytherapy-monotherapy groups. In this study, we planned to investigate whether the use of polytherapy has an effect on the increase in C-IMT and we obtained statistically significant results. In our study, we examined whether there was a significant difference in C-IMT between 30 healthy controls and 38 epilepsy patients, who were matched in terms of age, sex, and BMI. As a result, it was seen that there was a statistically significant C-IMT increase in the patient group compared to the control group (Table 1).

When we divided epilepsy patients as monotherapy and polytherapy, the increase in CIMT in the polytherapy group was statistically significant compared to the monotherapy group. However, when monotherapy and healthy control groups were compared, the result did not reach statistical significance (Table 2). In our study, the disease duration did not affect CIMT (Figure 1)

The results of C-IMT studies in epilepsy patients are controversial due to the diversity of patient groups (age, therapy, comparison vb.). In one study, while an increase in CIMT was observed in epilepsy patients compared to the healthy control group, the same study did not find a significant difference between patients taking valproic acid

\begin{tabular}{|c|c|c|c|c|c|c|c|}
\hline & Control (n:30) & Monotherapy (n:16) & Polytherapy (n:22) & $P$ values & P1 & P2 & P3 \\
\hline C-IMT & $0.37( \pm 0.04)$ & $0.40( \pm 0.08)$ & $0.46( \pm 0.08)$ & $<0.001$ & 0.35 & $<0.001$ & 0.01 \\
\hline Age (years) & $27.57( \pm 6.09)$ & $23.88( \pm 7.32)$ & $26.82( \pm 7.73)$ & 0.227 & - & - & - \\
\hline Female, n (\%) & $19(63.3 \%)$ & $10(62.5 \%)$ & $14(63.6 \%)$ & 0.997 & - & - & - \\
\hline BMI $\left(\mathrm{kg} / \mathrm{m}^{2}\right)$ & $24.16( \pm 3.52)$ & $22.44( \pm 4.28)$ & $23.91( \pm 4.44)$ & 0.371 & - & - & - \\
\hline \multicolumn{8}{|l|}{ Serum lipid level (mg) } \\
\hline LDL cholesterol & $99.62( \pm 14.20)$ & $103.06( \pm 30.51)$ & $111.18( \pm 30.64)$ & 0.275 & - & - & - \\
\hline HDL cholesterol & $53.82( \pm 14.46)$ & $58.44( \pm 13.92)$ & $58.44( \pm 13.93)$ & 0.162 & - & - & - \\
\hline Triglyceride & $109.08(33.28)$ & $93.00( \pm 33.23)$ & $105.9( \pm 35.83)$ & 0.629 & - & - & - \\
\hline Disease duration (years) & & $5.71( \pm 5.29)$ & $9.39( \pm 5.80)$ & 0.054 & & & \\
\hline
\end{tabular}


and enzyme-inducing drugs (16). In a meta-analysis of 15 studies involving 1175 epileptic patients, the majority of epilepsy patients using AEDs had increased C-IMT (15).

Among AEDs, especially CBZ and VA were more significantly associated with the increase in C-IMT. While phenytoin had no significant effect on C-IMT, studies about LMT were inconclusive. The results of phenytoin were attributed to study group smallness and heterogeneity. In our study, there was no difference between the groups in terms of lipid profile, and the increase in C-IMT was independent of lipid level. In the literature, some studies are compatible with this study $(10,17)$, as well as studies that correlate with lipid increase (18). The effect of treatment duration on CIMT is controversial. While studies are stating that the duration is independently effective on the increase in C-IMT $(18,19)$, there are also studies stating that it does not have any effect $(10,16,20)$. We could not evaluate the duration of treatment because we had polytherapy patients in the study group. We looked at the duration of epilepsy in our patients. In our study, the duration of epilepsy did not affect C-IMT.

When evaluating the increase in C-IMT in epilepsy patients, most of the studies included patients receiving monotherapy and analyses were made on specific drug groups. On the other hand, we divided the patients into monotherapy and polytherapy instead of a group using a certain drug. Similar to our study, Çalık et al. (18) made a distinction between monotherapy and polytherapy in pediatric patients and found the increase in C-IMT compatible in our study.

In addition, since epilepsy patients who did not use drugs were not included in the studies, it was interpreted that it could not be determined whether atherosclerosis was caused by the disease itself or the drugs used (16). When we separate and evaluate our patients as monotherapy and polytherapy, there is no difference between the monotherapy group and the control group in terms of C-IMT increase, but the fact that the polytherapy group is significant compared to the monotherapy group may highlight the effect of drugs rather than the disease. There is a need to work with larger patient groups for stronger comments on this issue. The limitations of our study are its cross-sectional nature and heterogeneous drug use in our patients.

\section{CONCLUSION}

According to our study results, it may be recommended to closely monitor atherosclerotic risk factors to prevent future vascular events, especially in epilepsy patients receiving polytherapy, however, more research is needed on this subject.

\section{ETHICAL DECLARATIONS}

Ethics Committee Approval: The study was approved by the Harran University Faculty of Medicine Ethics Committee (Date: 08.07.2019, Decision No: 07-13)

Informed Consent: All patients signed the free and informed consent form.

Referee Evaluation Process: Externally peer-reviewed.

Conflict of Interest Statement: The authors have no conflicts of interest to declare.

Financial Disclosure: The authors declared that this study has received no financial support.

Author Contributions: The authors declare that they have all participated in the design, execution, and analysis of the paper, and that they have approved the final version.

\section{REFERENCES}

1. Ngugi AK, Bottomley C, Kleinschmidt I, Sander JW, Newton CR. Estimation of the burden of active and lifetime epilepsy: a metaanalytic approach. Epilepsia 2010; 51: 883-90.

2. Shen C, Chen F, Zhang Y, Guo Y, Ding M. Association between use of antiepileptic drugs and fracture risk: a systematic review and meta-analysis. Bone 2014; 64: 246-53.

3. Eddy CM, Rickards HE, Cavanna AE. Behavioral adverse effects of antiepileptic drugs in epilepsy. J Clin Psychopharmacol 2012; 32: 362-75.

4. Mintzer S. Metabolic consequences of antiepileptic drugs. Curr Opin Neurol 2010; 23: 164-69.

5. Perucca P, Gilliam FG. Adverse effects of antiepileptic drugs. Lancet Neurol 2012; 11: 792-02

6. Chang CS, Liao CH, Lin CC, Lane HY, Sung FC, Kao CH. Patients with epilepsy are at an increased risk of subsequent stroke: a population-based cohort study. Seizure 2014; 23: 377-81.

7. Hsieh CY, Lai EC, Yang YH, Lin SJ. Comparative stroke risk of antiepileptic drugs in patients with epilepsy. Epilepsia 2013; 54: 172-80.

8. Mahmoudian T, Iranpour R, Messri N. Serum lipid levels during carbamazepine therapy in epileptic children. Epilepsy Behav 2005; 6: 257-9.

9. Karabiber H, Sonmezgoz E, Ozerol E, Yakinci C, Otlu B, Yologlu S. Effects of valproate and carbamazepine on serum levels of homocysteine, vitamin B12, and folic acid. Brain Dev 2003; 25: 113-5.

10. Talaat FM, Kamel T, Rabah AM, Ahmed SM, El-Jaafary SI, Abdelaziz GH. Epilepsy and antiepileptic drugs: risk factors for atherosclerosis. Int J Neurosci 2015; 125: 507-11.

11. Hamed SA. Atherosclerosis in epilepsy: its causes and implications. Epilepsy Behav 2014; 41: 290-96.

12. Polak JF, Pencina MJ, Pencina KM, O'Donnell CJ, Wolf PA, D'Agostino RB Sr. Carotid-wall intima-media thickness and cardiovascular events. New Engl J Med 2011; 21; 365: 213-21.

13. Hermann DM, Gronewold J, Lehmann N, et al. Intima-media thickness predicts stroke risk in the Heinz Nixdorf Recall study in association with vascular risk factors, age and gender. Atherosclerosis 2012; 224: 84-9.

14. Scheffer IE, Berkovic S, Capovilla G, et al. ILAE classification of the epilepsies: position paper of the ILAE Commission for classification and terminology. Epilepsia 2017; 58: 512-21. 
15.Lai Q, Shen C, Zheng Y, Zhang Y, Guo Y, Ding M. Effects of antiepileptic drugs on the carotid artery intima-media thickness in epileptic patients. J Clin Neurol 2017; 13: 371-9.

16. Mehrpour M, Shojaie M, Zamani B, Gharibzadeh S, Abbasi M. Atherogenic consequence of antiepileptic drugs: a study of intima-media thickness. Neurol Sci 2014; 35: 253-7.

17. Karatoprak E, Tosun O. Effects of valproic acid and levetiracetam monotherapy on carotid intima-media and epicardial adipose tissue thickness in non-obese children with epilepsy. Brain Dev 2020; 42: 165-70.

18. Calik M, Ozkan HY, Ethemoglu O, et al. The measurement of both carotid intima-media thickness and epicardial adipose tissue thickness in children with epilepsy receiving antiepileptic drug therapy. Epilepsy Behav 2018; 85: 110-4.

19. Tan TY, Lu CH, Chuang HY, et al. Long-term antiepileptic drug therapy contributes to the acceleration of atherosclerosis. Epilepsia 2009; 50: 1579-86.

20.Erdemir A, Cullu N, Yiş U, et al. Evaluation of serum lipids and carotid artery intima-media thickness in epileptic children treated with valproic acid. Brain Dev 2009; 31: 713-6. 